\title{
A RECONFIGURABLE COMPLIANT FOUR-BAR MECHANISM WITH MULTIPLE OPERATION MODES
}

\author{
Abhilash Nayak \\ École Centrale de Nantes \\ Laboratoire des Sciences du Numérique de Nantes (LS2N)* \\ UMR CNRS 6004 \\ 1 rue de la Noë 44321 Nantes, France. \\ Email: Abhilash.Nayak@Is2n.fr
}

\author{
Guangbo $\mathrm{Hao}^{\dagger}$ \\ School of Engineering- \\ Electrical and Electronic Engineering \\ University College Cork, Cork, Ireland \\ E-mail: G.Hao@ucc.ie
}

\author{
Haiyang Li \\ School of Engineering- \\ Electrical and Electronic Engineering \\ University College Cork, Cork, Ireland \\ E-mail: Haiyang.Li@umail.ucc.ie
}

\author{
Stéphane Caro \\ CNRS, Laboratoire des Sciences du \\ Numérique de Nantes (LS2N), UMR CNRS 6004 \\ 1 rue de la Noë, 44321 Nantess, France. \\ Email: Stephane.Caro@Is2n.fr
}

\section{ABSTRACT}

Although reconfigurable rigid-body mechanisms have been extensively studied over two decades, their compliant counterparts have not received the similar attention yet. This paper aims to design a reconfigurable compliant four-bar mechanism with multiple operation modes. A planar equilateral four-bar mechanism is considered at its constraint singularity. The multiple operation modes of this linkage are kinematically exploited to design a reconfigurable compliant four-bar mechanism, which generates rotational or translational motions based on two actuated joints. Simulation is conducted to investigate the comprehensive kinematics of the reconfigurable compliant mechanism. A 3D printed prototype of the novel reconfigurable compliant mechanism at hand is presented.

\section{INTRODUCTION}

Although mechanisms are often composed of rigid bodies connected by joints, compliant mechanisms include flexible el-

\footnotetext{
${ }^{*}$ IRCCyN (Research Institute in Communications and Cybernetics of Nantes) merged with LINA (Computer Science Laboratory of Nantes) to give birth to LS2N (Digital Science Laboratory of Nantes) on January $1^{\text {st }}, 2017$

$\dagger$ Address all correspondence to this author.
}

ements whose elastic deformation is utilized in order to transmit a force and/or motion. There are different ways to design compliant mechanisms, such as the kinematic based approaches, the building blocks approaches, and the structural optimizationbased approaches [1-4]. In the kinematic based approach, the joints of a chosen rigid-body mechanism are replaced by appropriate compliant joints followed by pseudo-rigid body modeling [2-4]. This method is advantageous due to the extensive choice of existing rigid-body mechanisms and their modeling tools. Parallel or closed-loop rigid-body architectures gain an upper hand here as their intrinsic properties favour the characteristics of compliant mechanisms like compactness, symmetry to reduce parasitic motions, low stiffness along the desired degrees of freedom (DOF) and high stiffness in other directions. Moreover, compliant mechanisms usually work around a given position for small range of motions and hence they can be designed by considering existing parallel manipulators in parallel singular configurations. Parallel singularity can be an actuation singularity, constraint singularity or a compound singularity as explained in [5-7]. Rubbert et al. used an actuation singularity to type-synthesize a compliant medical device $[8,9]$. Another interesting kind of parallel singularity for a parallel manipulator that does not depend on the choice of ac- 


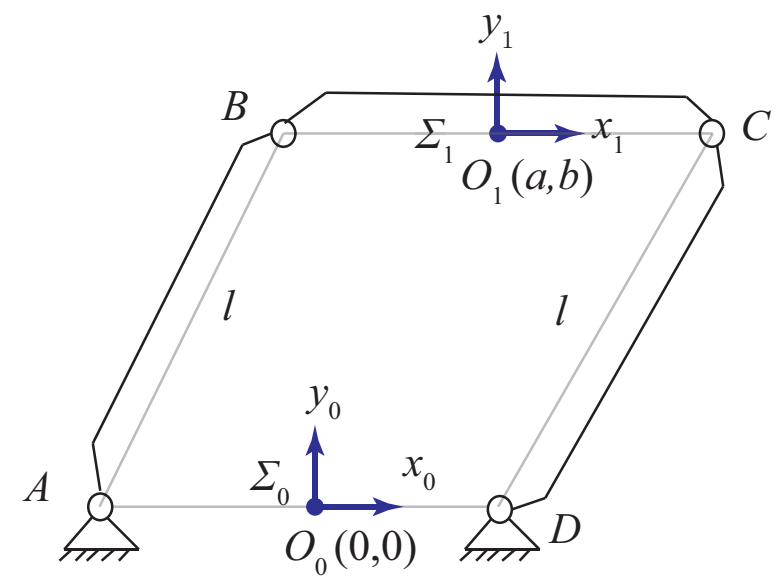

FIGURE 1: AN EQUILATERAL FOUR BAR LINKAGE.

tuation is a constraint singularity [10]. It divides the workspace of a parallel manipulator into different operation modes resulting in a reconfigurable mechanism. Algebraic geometry tools have proved to be efficient in performing global analysis of parallel manipulators and recognizing their operation modes leading to mobility-reconfiguration [11-13]. Though there are abundant reconfigurable rigid-body mechanisms in the literature, the study of reconfigurable compliant mechanisms is limited. Hao studied the mobility and structure reconfiguration of compliant mechanisms [14] while Hao and Li introduced a position-spacebased structure reconfiguration (PSR) approach to the reconfiguration of compliant mechanisms and to minimize parasitic motions $[15,16]$. In this paper, one of the simplest yet ubiquitous parallel mechanisms, a planar equilateral four-bar linkage is considered at a constraint singularity configuration to synthesize a reconfigurable compliant four-bar mechanism. From our best understanding, this is the first piece of work that considers a constraint singularity to design a reconfigurable compliant mechanism with multiple operation modes, also called motion modes. This paper is organized as follows : Kinematic analysis of a rigid four-bar mechanism is performed to determine the constraint singularities and different operation modes. Rigid-body replacement design approach is followed to further synthesize a reconfigurable compliant four-bar mechanism and the motion type associated to each operation mode is verified through non-linear Finite Element Analysis (FEA).

\section{KINEMATIC ANALYSIS AND OPERATION MODES OF A FOUR BAR LINKAGE}

A planar equilateral four-bar linkage with equal link lengths, $l$ is depicted in Fig. 1. Link $A D$ is fixed, $A B$ and $C D$ are the cranks and $B C$ is the coupler. Origin of the fixed frame $\left(\Sigma_{0}\right), O_{0}$ coincides with the center of link $A D$ while that of the moving frame $\left(\Sigma_{1}\right) O_{1}$ with the center of $B C$. The coordinate axes are oriented in such a way that the position vectors of the intersection points between the revolute joint axes and the $x_{0} y_{0}$ plane can be homogeneously written as follows:

$$
\begin{array}{ll}
\mathbf{r}_{A}^{0}=\left[1, \frac{-l}{2}, 0\right]^{T} & \mathbf{r}_{D}^{0}=\left[1, \frac{l}{2}, 0\right]^{T} \\
\mathbf{r}_{B}^{1}=\left[1, \frac{-l}{2}, 0\right]^{T} & \mathbf{r}_{C}^{1}=\left[1, \frac{l}{2}, 0\right]^{T}
\end{array}
$$

The displacement of the coupler with respect to the fixed frame can be rendered by $(a, b, \phi)$, where $a$ and $b$ represent the positional displacement of the coupler (nothing but the coordinates of point $O_{1}$ in $\left.\Sigma_{0}\right)$ and $\phi$ is the angular displacement about $z_{0}$-axis (angle between $x_{0}$ and $x_{1}$ ). Thus, the corresponding set of displacements can be mapped onto a three-dimensional projective space, $\mathbb{P}^{3}$ with homogeneous coordinates $x_{i}(i=1,2,3,4)$ [17]. This mapping (also known as Blashke mapping in the literature) is defined by the following matrix $\mathrm{M}$ :

$$
\mathbf{M}=\left[\begin{array}{ccc}
1 & 0 & 0 \\
\frac{2 x_{1} x_{3}+2 x_{2} x_{4}}{x_{3}^{2}+x_{4}^{2}} & \frac{-x_{3}^{2}+x_{4}^{2}}{x_{3}^{2}+x_{4}^{2}} & \frac{-2 x_{3} x_{4}}{x_{3}^{2}+x_{4}^{2}} \\
\frac{-2 x_{1} x_{4}+2 x_{2} x_{3}}{x_{3}^{2}+x_{4}^{2}} & \frac{2 x_{3} x_{4}}{x_{3}^{2}+x_{4}^{2}} & \frac{-x_{3}^{2}+x_{4}^{2}}{x_{3}^{2}+x_{4}^{2}}
\end{array}\right]
$$

The planar kinematic mapping can also be derived as a special case of Study's kinematic mapping by equating some of the Study parameters to zero [11]. To avoid the rotational part of $\mathbf{M}$ to be undefined, the following equation is defined:

$$
H:=x_{3}^{2}+x_{4}^{2}=1
$$

Without loss of generality, $x_{i}$ can be expressed in terms of $(a, b, \phi)$, as follows [17] :

$$
\begin{aligned}
& x_{1}: x_{2}: x_{3}: x_{4}=(a u-b v):(a v+b u): 2 u: 2 v \\
& \text { with } \quad u=\sin \left(\frac{\phi}{2}\right), \quad v=\cos \left(\frac{\phi}{2}\right)
\end{aligned}
$$

\section{Constraint Equations}

Points $B$ and $C$ are constrained to move along circles of centers $A$ and $D$, respectively and with radius $l$ each. The position vectors of points $B$ and $C$ are expressed algebraically in frame $\Sigma_{0}$ as follows :

$$
\mathbf{r}_{B}^{0}=M \mathbf{r}_{B}^{1} \quad ; \quad \mathbf{r}_{C}^{0}=M \mathbf{r}_{C}^{1}
$$




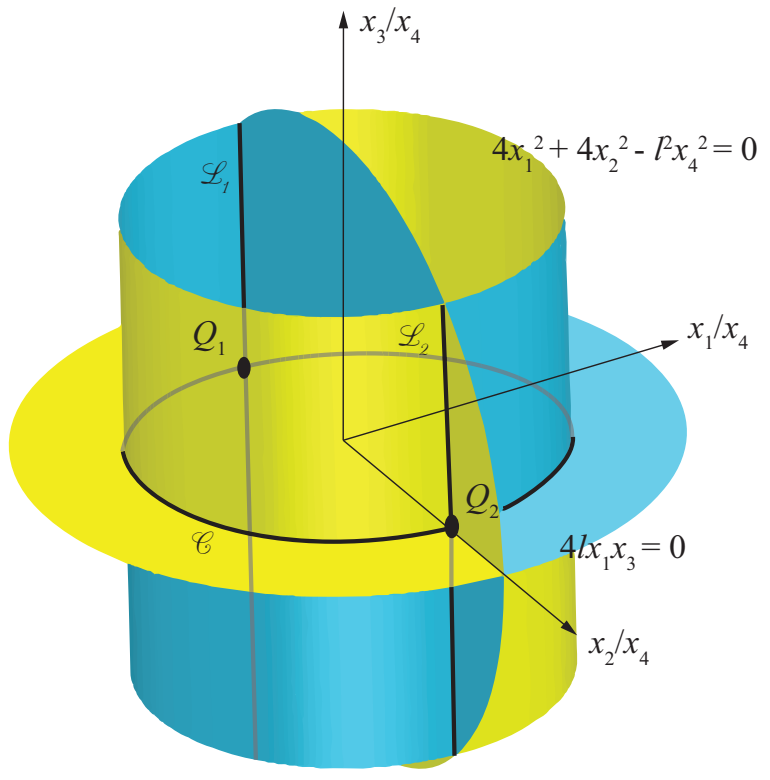

FIGURE 2: CONSTRAINT MANIFOLDS OF THE FOUR BAR LINKAGE IN IMAGE SPACE.

Therefore, the algebraic constraint equations take the form :

$$
\begin{aligned}
& \left(\mathbf{r}_{B}^{0}-\mathbf{r}_{A}^{0}\right)^{T}\left(\mathbf{r}_{B}^{0}-\mathbf{r}_{A}^{0}\right)=l^{2} \\
& \Longrightarrow g_{1}:=4\left(x_{1}^{2}+x_{2}^{2}\right)+4 l x_{1} x_{3}-l^{2} x_{4}^{2}=0 \\
& \left(\mathbf{r}_{C}^{0}-\mathbf{r}_{D}^{0}\right)^{T}\left(\mathbf{r}_{C}^{0}-\mathbf{r}_{D}^{0}\right)=l^{2} \\
& \Longrightarrow g_{2}:=4\left(x_{1}^{2}+x_{2}^{2}\right)-4 l x_{1} x_{3}-l^{2} x_{4}^{2}=0
\end{aligned}
$$

Since $g 1 \pm g 2=0$ gives the same variety, the final simplified constraint equations are :

$$
\begin{aligned}
& H_{1}:=g_{1}-g_{2}:=4 l x_{1} x_{3}=0 \\
& H_{2}:=g_{1}+g_{2}:=4\left(x_{1}^{2}+x_{2}^{2}\right)-l^{2} x_{4}^{2}=0
\end{aligned}
$$

Equation (9) degenerates into two planes $x_{1}=x_{3}=0$ into the image space and Eqn. (10) amounts to a cylinder with a circular cross-section in the image space. Assuming $x_{4} \neq 0$, these constraint manifolds can be represented in the affine space, $\mathbb{A}^{3}$, as shown in Fig. 2.

\section{Operation Modes}

The affine variety of the polynomials $H_{1}$ and $H_{2}$ amounts to all the possible displacements attainable by the coupler. This variety is nothing but the intersection of these constraint surfaces in the image space [11]. The intersections can be seen as two lines and a circle in Fig. 2. In fact, these curves can be algebraically represented by decomposing the constraint equations (9) and (10). A primary decomposition of the ideal $\mathfrak{I}=$ $\left\langle H_{1}, H_{2}\right\rangle$ onto the field $\mathbb{K}\left(x_{1}, x_{2}, x_{3}, x_{4}\right)$ results in the following sub-ideals:

$$
\begin{aligned}
& \mathfrak{I}_{1}=\left\langle x_{1}, 2 x_{2}-l x_{4}\right\rangle \\
& \mathfrak{I}_{2}=\left\langle x_{1}, 2 x_{2}+l x_{4}\right\rangle \\
& \mathfrak{I}_{3}=\left\langle x_{3}, 4\left(x_{1}^{2}+x_{2}^{2}\right)-l^{2} x_{4}^{2}\right\rangle
\end{aligned}
$$

It shows that this four-bar linkage has three operation modes. The Hilbert dimension of the ideals $\mathfrak{I}_{i}$ including the polynomial $H$ from Eqn. (4) is calculated to be one, indicating that the DOF of the four-bar mechanism is one in each of these three operation modes. $\mathfrak{I}_{1}$ and $\mathfrak{I}_{2}$ correspond to $x_{1}=0$ implying $u=\frac{b}{a}$ from Eqn. (5). Furthermore, for $\mathfrak{I}_{1}$, eliminating $u$ from $2 x_{2}-l x_{4}=0$ gives

$$
a^{2}+b^{2}-a l=0
$$

which is the equation of a circle of center point $B$ of Cartesian coordinates $\left(\frac{l}{2}, 0\right)$ and radius $\frac{l}{2}$ as shown in Fig. 3 .

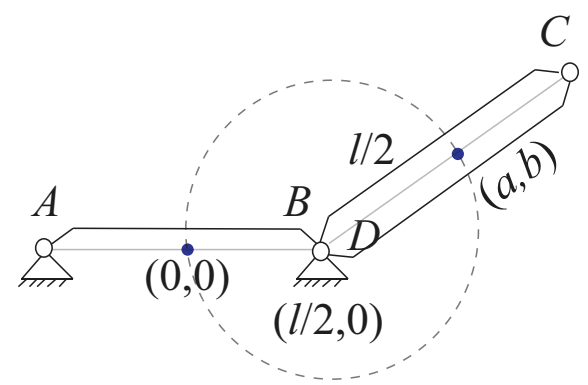

FIGURE 3: OPERATION MODE $1: a^{2}+b^{2}-a l=0$

Similarly, $\mathfrak{I}_{2}$ yields

$$
a^{2}+b^{2}+a l=0
$$

which is the equation of a circle of center point $C$ of Cartesian coordinates $\left(-\frac{l}{2}, 0\right)$ and radius $\frac{l}{2}$ as shown in Fig. 4.

The third ideal $\mathfrak{I}_{3}$ corresponds to $x_{3}=0$ and hence $u=0$ implying $\phi=0$. The second equation of the same ideal results in

$$
a^{2}+b^{2}-l^{2}=0
$$




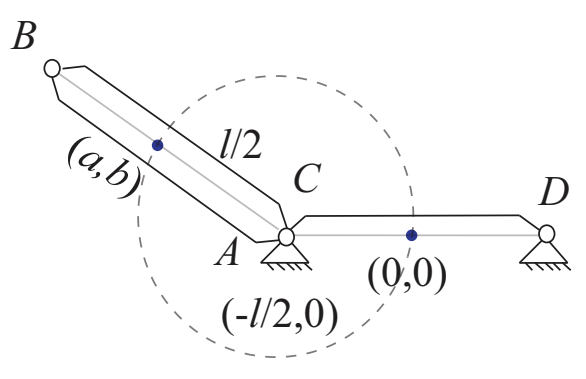

FIGURE 4: OPERATION MODE $2: a^{2}+b^{2}+a l=0$.

being the equation of a circle of center $(0,0)$ and radius $l$ as shown in Fig. 5. As a result, $\mathfrak{I}_{1}$ and $\mathfrak{I}_{2}$ represent rotational modes while $\mathfrak{I}_{3}$ represents a translational mode.

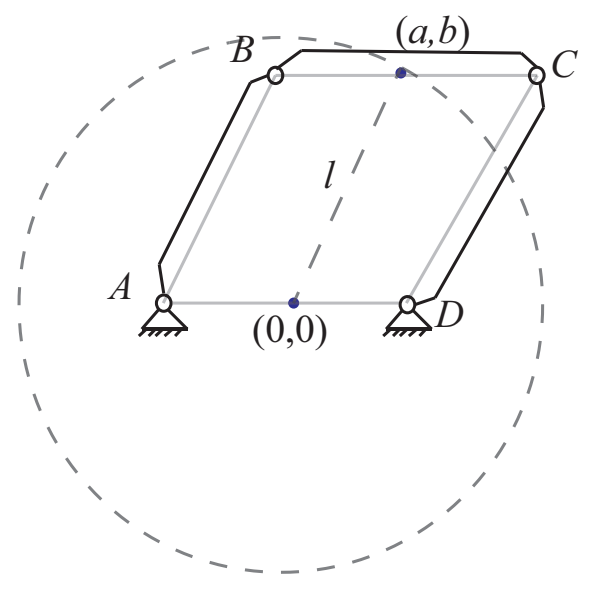

FIGURE 5: OPERATION MODE $3: a^{2}+b^{2}-l^{2}=0$.

Ultimately, in Fig. 2, the intersection lines $\mathscr{L}_{1}$ and $\mathscr{L}_{2}$ of the constraint manifolds portray the rotational motion modes while the circle $\mathscr{C}$ portrays the translational motion mode.

\section{Constraint Singularities}

These operation modes are separated by two similar constraint singularities shown in Fig. 6.

They can be algebraically represented by $x_{1}=x_{3}=4 x_{2}^{2}-$ $l^{2} x_{4}^{2}=0$. From Eqn. (5), these singularities occur when $b=0$, $\phi=0$ and $a= \pm l$. These two configurations correspond to the two points $Q_{1}$ and $Q_{2}$ in the image space shown in Fig. 2. At a constraint singularity, any mechanism gains one or more degrees of freedom. Therefore, in case of the four-bar linkage with equal link lengths, the DOF at a constraint singularity is equal 2. In this configuration, points $A, B, C$ and $D$ are collinear and the corresponding motion type is a translational motion along the
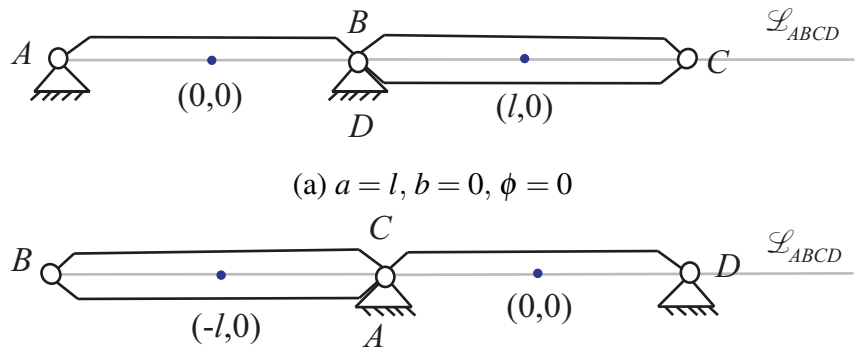

(b) $a=-l, b=0, \phi=0$

FIGURE 6: CONSTRAINT SINGULARITIES OF THE FOUR BAR MECHANISM.

normal to the line $\mathscr{L}_{A B C D}$ passing through the four points $A, B$, $C$ and $D$ combined with a rotation about an axis directed along $z_{0}$ and passing through $\mathscr{L}_{A B C D}$. Eventually, it is noteworthy that two actuators are required in order to control the end-effector in those constraint singularities in order to manage the operation mode changing.

\section{DESIGN AND ANALYSIS OF A COMPLIANT FOUR-BAR MECHANISM}

In this section, two compliant four-bar mechanisms, compliant four-bar mechanism-1 and compliant four-bar mechanism-2, are proposed based on the operation modes and constraint singularities of the four-bar rigid-body mechanism shown in Fig. 6b. Moreover, the desired motion characteristics of the compliant four-bar mechanism-2 are verified by nonlinear FEA simulations.

\section{Design of a compliant four-bar mechanism}

Based on the constraint singularity configuration of the four-bar rigid-body mechanism represented in Fig. 6, a compliant four-bar mechanism can be designed through kinematically replacing the rigid rotational joints with compliant rotational joints [15]. Each of the compliant rotational joints can be any type compliant rotational joint such as cross-spring rotational joint, notch rotational joint and cartwheel rotational joint [3]. As shown in Fig. 7, a compliant four-bar mechanism, termed as the compliant four-bar mechanism-1, has been designed by replacing the four rigid rotational joints with three cross-spring rotational joints (RJ-0, RJ-1 and RJ-3) and one leaf-type isoscelestrapezoidal rotational joint that provides remote rotation centre (RJ-2).

For small motion ranges, the compliant four-bar mechanism- 1 has the same operation modes as the fourbar rigid-body mechanism shown in Fig. 6, via controlling the rotations of the Bar-1 and Bar-3. Moreover, both the compliant four-bar mechanism-1 and the four-bar rigid-body 

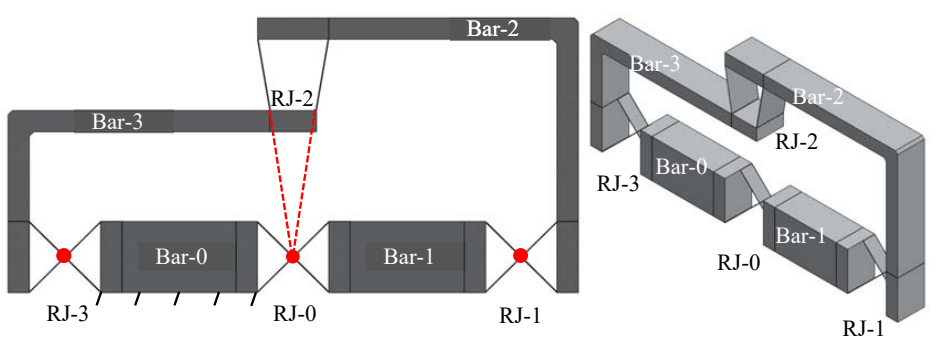

(a)

\section{FIGURE 7: COMPLIANT FOUR-BAR MECHANISM-1}
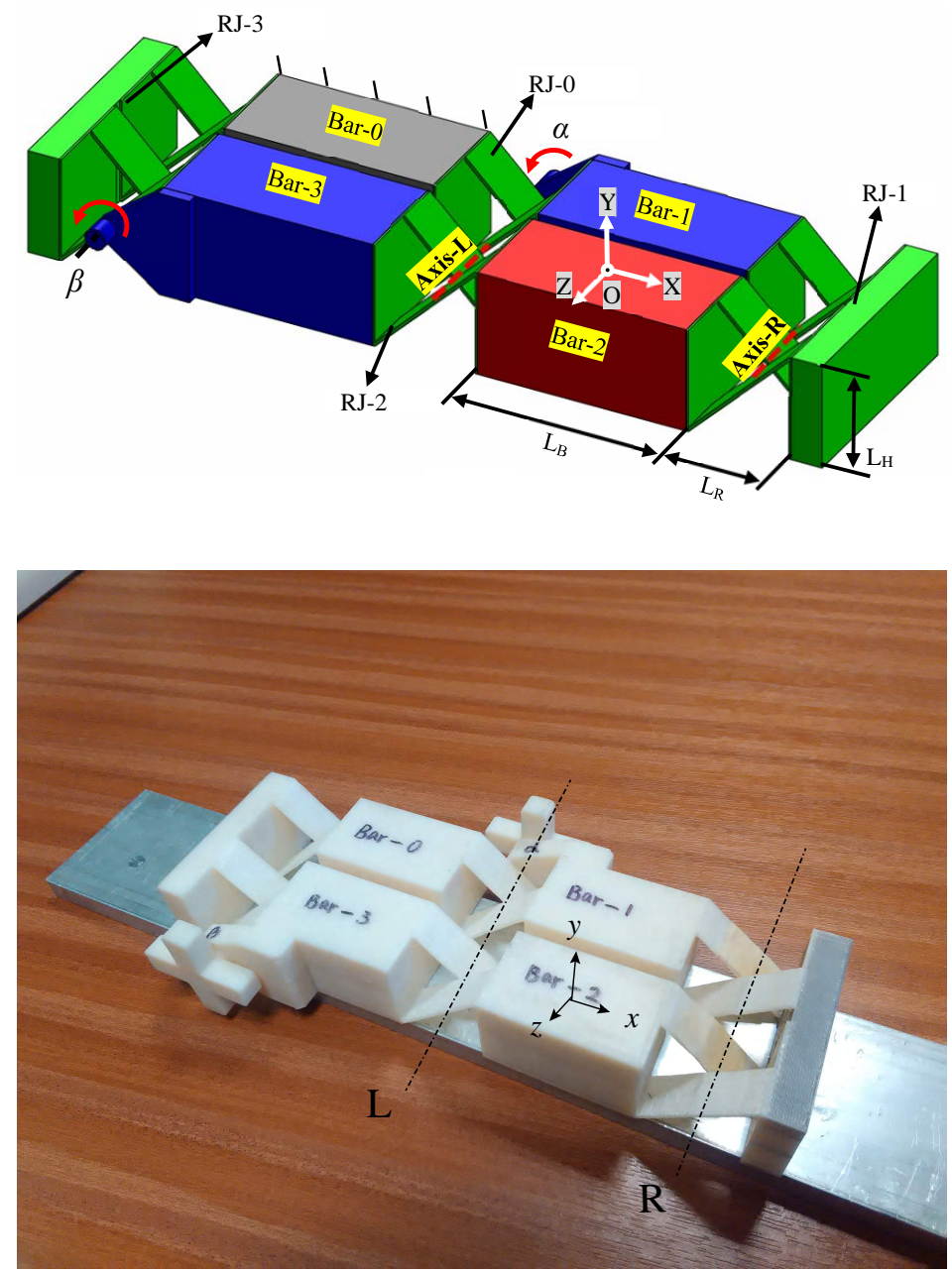

FIGURE 8: CAD MODEL AND PROTOTYPE OF THE COMPLIANT FOUR-BAR MECHANISM-2 mechanism are plane motion mechanisms. Additionally, the three cross-spring rotational joints in the compliant four-bar mechanism-1 can be replaced by other types of rotational joints, which can form different compliant four-bar mechanisms. In this paper, cross-spring rotational joints are employed due to their large motion ranges while small rotation centre shifts. However, the leaf-type isosceles-trapezoidal rotational joint in the compliant four-bar mechanism-1 performs larger rotation centre shifts compared with the cross-spring rotational joint. Therefore, the compliant four-bar mechanism- 1 can be improved by replacing the leaf-type isosceles-trapezoidal rotational joint with a cross-spring rotational joint. Such an improved design can be seen in Fig. 8, which is termed as the compliant four-bar mechanism-2. Note that, in Fig. 8, the RJ-0 and RJ-2, are traditional cross-spring rotational joints, while both the RJ-1 and the RJ-3 are double cross-spring joints introduced in this paper. Each of the rotational joints, RJ-1 and RJ-3, consists of two traditional cross-spring rotational joints in series. We specify that the Bar-0 is fixed to the ground and the Bar-2 is the output motion stage, also named coupler. The main body including rigid bars and compliant joints of the proposed compliant four-bar mechanism-2 can be fabricated monolithically using a CNC milling machine. It can also be 3D printed, and a 3Dprinted prototype is shown in Fig. 8. The bars of the prototype have many small through holes, which can reduce material consumption and improve dynamic performance. Additionally, two cross-shaped parts are added to the actuated bars, which are used to actuate the mechanism by hands. The operation modes of the compliant four-bar mechanism-2 as output stage are analyzed in the following sections.

\section{OPERATION MODES OF THE COMPLIANT FOUR-BAR MECHANISM-2}

Like the four-bar rigid-body mechanism shown in Fig. 6b, the output motion stage (Bar 2) of the compliant four-bar mechanism-2 has multiple operation modes under two rotational actuations (controlled by input displacements $\alpha$ and $\beta$ ), as shown in Fig. 8. However, the compliant four-bar mechanism-2 has more operation modes than the rigid counterpart. In order to simplify the analysis, let $\alpha$ and $\beta$ be non-negative. A coordinate system is defined in Fig. 8, which is located on Bar 2. Based on this assumption, operation modes of the compliant four-bar mechanism-2 are listed below :

1. Operation mode I : Rotation in the XY-plane about the Axis$\mathrm{L}$, when $\alpha>0$ and $\beta=0$, as shown in Fig. 9a,

2. Operation mode II : Rotation in the XY-plane about the Axis- $\mathrm{R}$ when $\alpha=0$ and $\beta>0$, as shown in Fig. $9 \mathrm{~b}$,

3. Operation mode III : Rotation in the XY-plane about other axes except the Axis-L and Axis- $\mathrm{R}$, when $\alpha \neq \beta>0$, as shown in Fig. 9c, and

4. Operation mode IV : Pure translations in the XY-plane along 

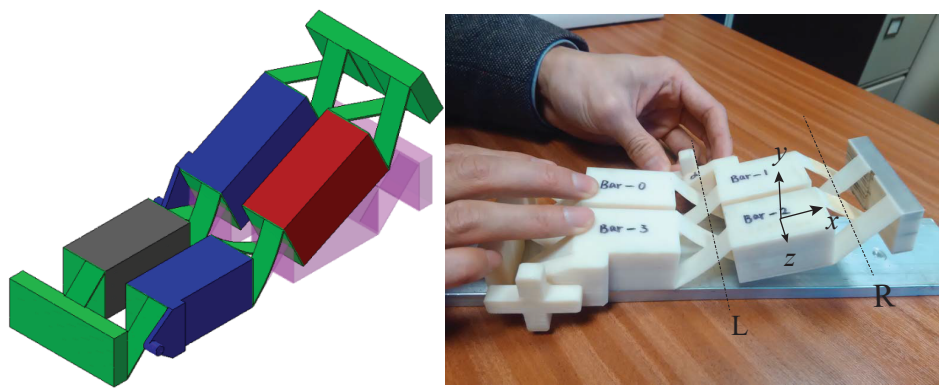

(a) Operation mode I : Rotation in the XY-plane about the Axis-L

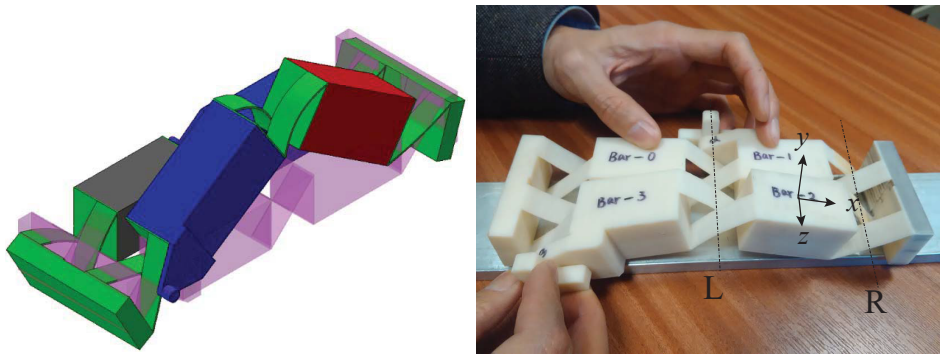

(b) Operation mode II : Rotation in the XY-plane about the Axis-R
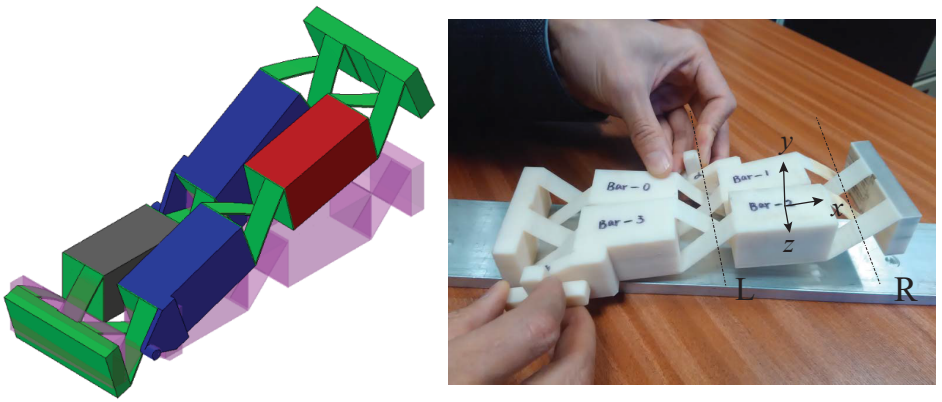

(c) Operation mode III : Rotation in the XY-plane about other axes except the Axis-L and Axis- $\mathrm{R}$
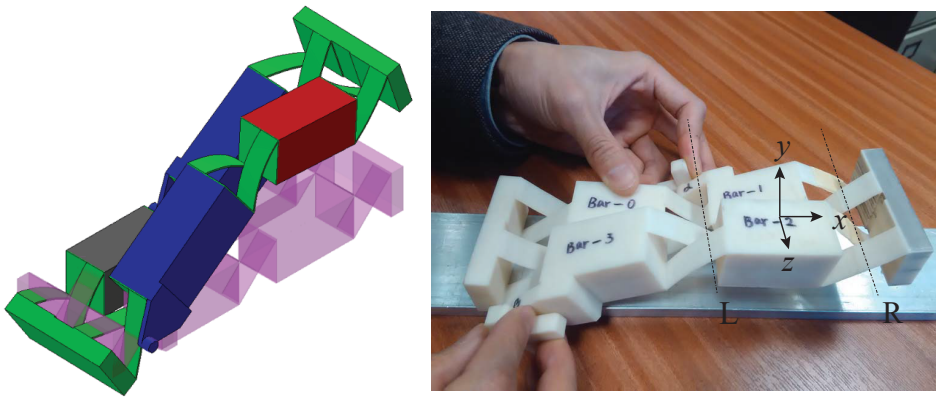

(d) Operation mode IV : Pure translations in the XY-plane along the $\mathrm{X}$ and $\mathrm{Y}$ axes

FIGURE 9: OPERATION MODES OF THE COMPLIANT FOUR-BAR MECHANISM-2 the $\mathrm{X}$ - and Y-axes, when $\alpha=\beta>0$, as shown in Fig. 9d.

These operation modes are also highlighted through the printed prototype in Fig. 9. The primary motions of output motion stage (Bar-2) are the rotation in the XY plane and the translations along the $\mathrm{X}$-and Y-axes; while the rotations in the $\mathrm{XZ}$ and $\mathrm{YZ}$ planes and translational motion along the $\mathrm{Z}$-axis are the parasitic motions that are not the interest of this paper. Moreover, the rotation angle in the XY-plane and the Y-axis translational motion can be estimated analytically using Eqs. (17) and (18). However, the $\mathrm{X}$-axis translational motion cannot be accurately estimated in such a simple way, because it is heavily affected by the shift of the rotation centres of the two cross-spring rotational joints [18]. The $\mathrm{X}$-axis translational motion will be analytically studied in our future work, but will be captured by non-linear FEA.

$$
\begin{aligned}
\theta_{Z} & =\alpha-\beta \\
D_{Y} & =\frac{1}{2}\left(L_{B}+L_{R}\right)(\sin \alpha+\sin \beta)
\end{aligned}
$$

where $\theta_{Z}$ is the rotation in the $\mathrm{XY}$ plane and $D_{Y}$ is the translational displacement in the Y-axis. $L_{B}$ and $L_{R}$ are the geometrical dimensions of the reconfigurable mechanism at hand, as defined in Fig. 8.

\section{SIMULATIONS OF THE OPERATION MODES}

In order to verify the operation modes of the $4 \mathrm{R}$ compliant mechanism-2, nonlinear FEA software is employed to simulate the motions of the compliant four-bar mechanism-2. For the FEA simulations, let $L_{B}$ be $100 \mathrm{~mm}, L_{R}$ and $L_{H}$ be $50 \mathrm{~mm}$, the beam thickness be $1 \mathrm{~mm}$, the beam width be $23 \mathrm{~mm}$, the Poissons ratio be 0.33 , and the Youngs modulus be $6.9 \mathrm{GPa}$. Commercial software, COMSOL MULTIPHYSICS, is selected for the nonlinear FEA simulations, using the 10-node tetrahedral element and finer meshing technology (minimum element size $0.2 \mathrm{~mm}$, curvature factor 0.4 , and resolution of narrow regions 0.7 ). Note that the translational displacements of the Bar-2 along the $\mathrm{X}$ and $\mathrm{Y}$ axes are measured at the centre point of the top surface of the Bar-2 (termed as the interest point), as shown in Fig. 8. Results of the simulations are plotted in Figs. 10 to 13, and the following conclusions are drawn :

1. The maximum difference between the FEA results and the analytical results in terms of the Y-axis translation of the interest point (the centre of the top surface of the Bar-2) is tiny, which is less than $0.5 \%$ as shown in Figs. 10a, 11a, 12a and 13a.

2. The FEA results of the rotation in the XY-plane match the analytical results very well. The difference is less than $0.8 \times$ $10^{-3} \mathrm{rad}(0.5 \%$ of the maximum rotation angle), which is shown in Figs. 10b, 11b and 12b. 


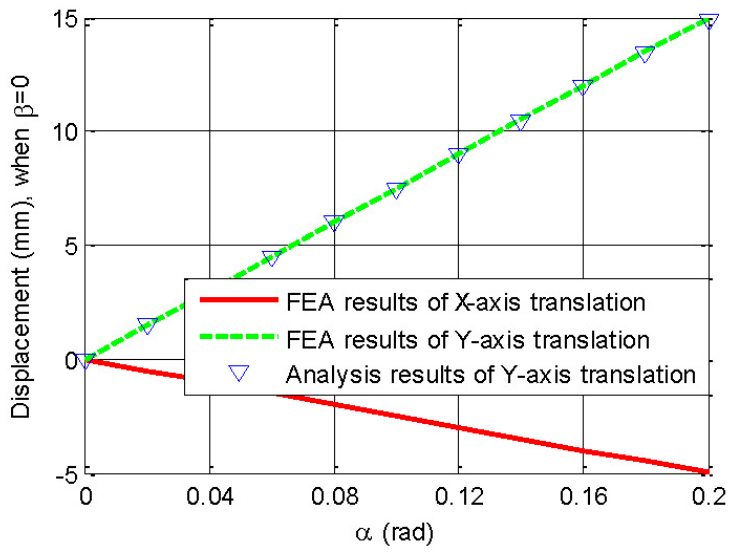

(a) Translations along the $\mathrm{X}$ and $\mathrm{Y}$ axes

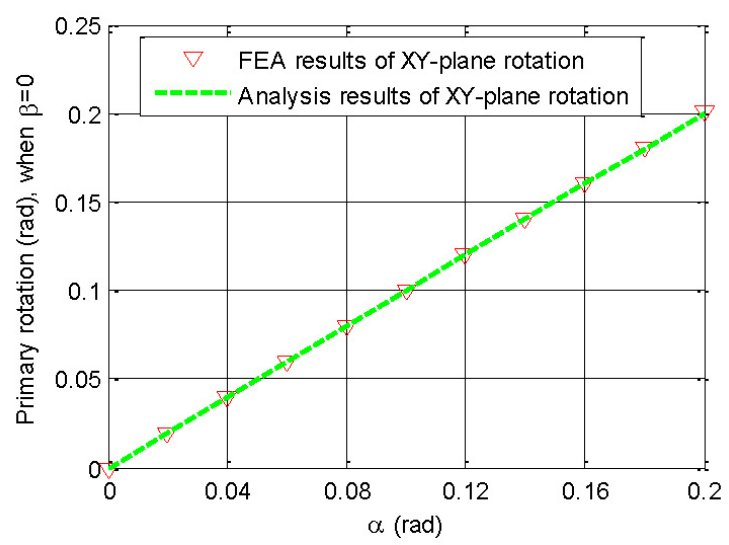

(b) Rotation about the Axis-L

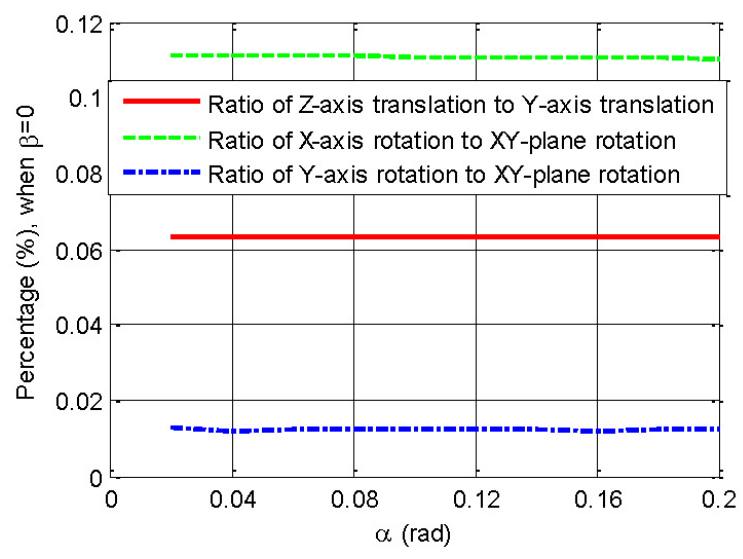

(c) parasitic motions (rotations about the $\mathrm{X}$ - and Y-axes and translation along the Z-axis)

FIGURE 10: FEA RESULTS FOR OPERATION MODE I

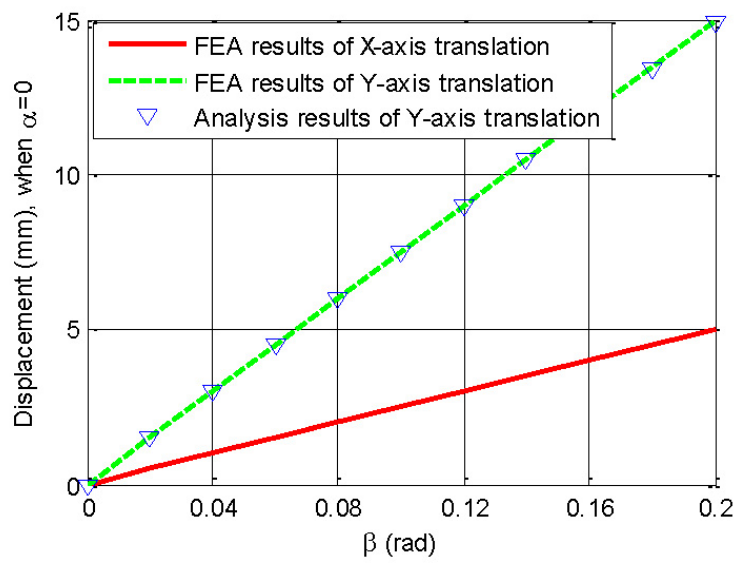

(a) Translations along the $\mathrm{X}$ and $\mathrm{Y}$ axes

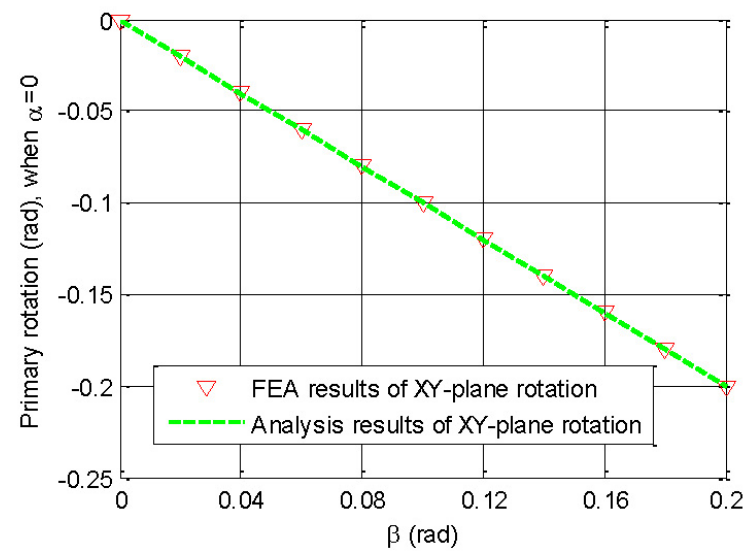

(b) Rotation about the Axis-L

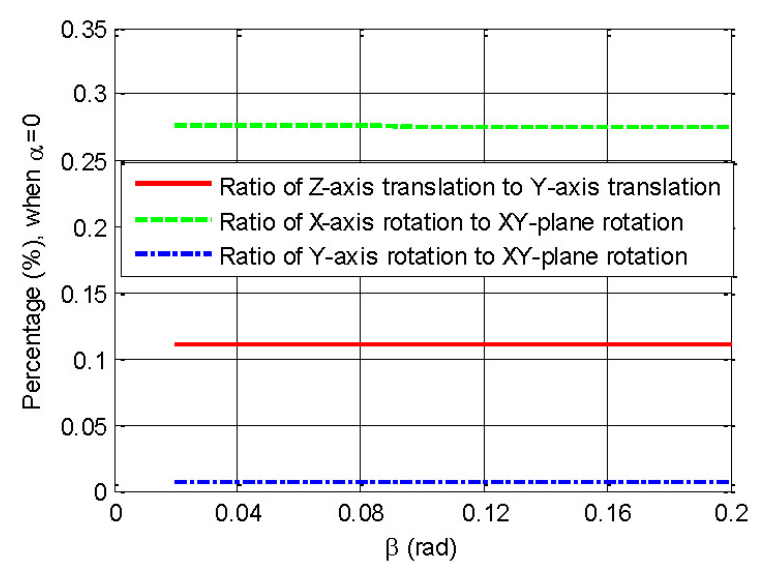

(c) parasitic motions (rotations about the $\mathrm{X}$ - and Y-axes and translation along the Z-axis)

FIGURE 11: FEA RESULTS FOR OPERATION MODE II 


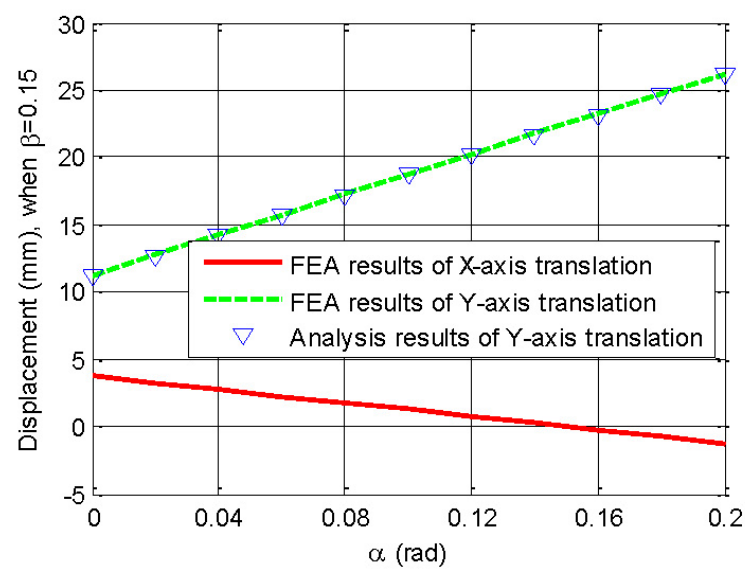

(a) Translations along the $\mathrm{X}$ and $\mathrm{Y}$ axes

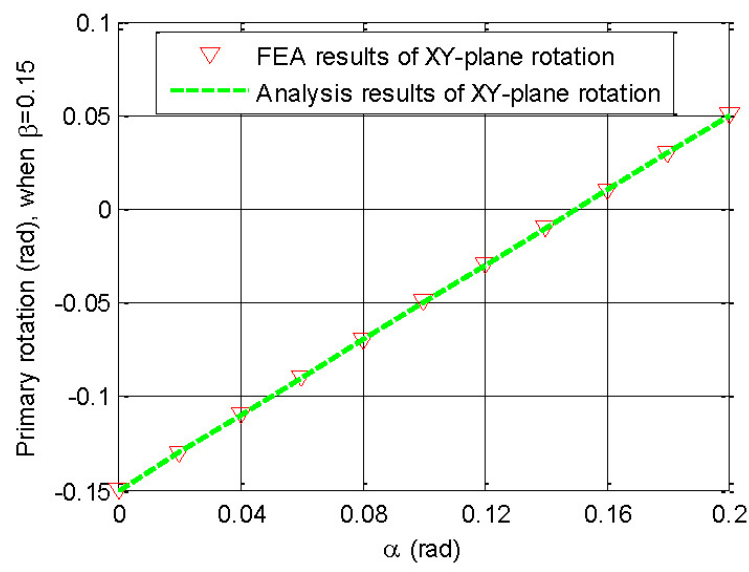

(b) Rotation about the Axis-L

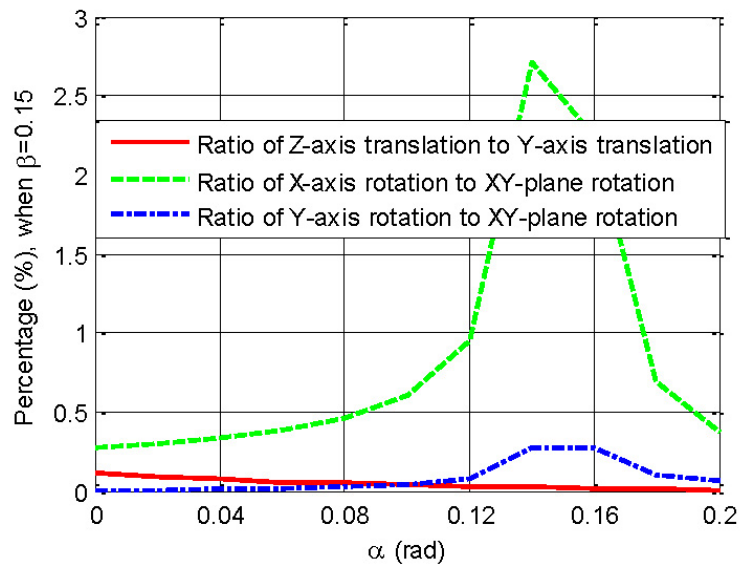

(c) parasitic motions (rotations about the $\mathrm{X}$ - and $\mathrm{Y}$-axes and translation along the Z-axis)

FIGURE 12: FEA RESULTS FOR OPERATION MODE III

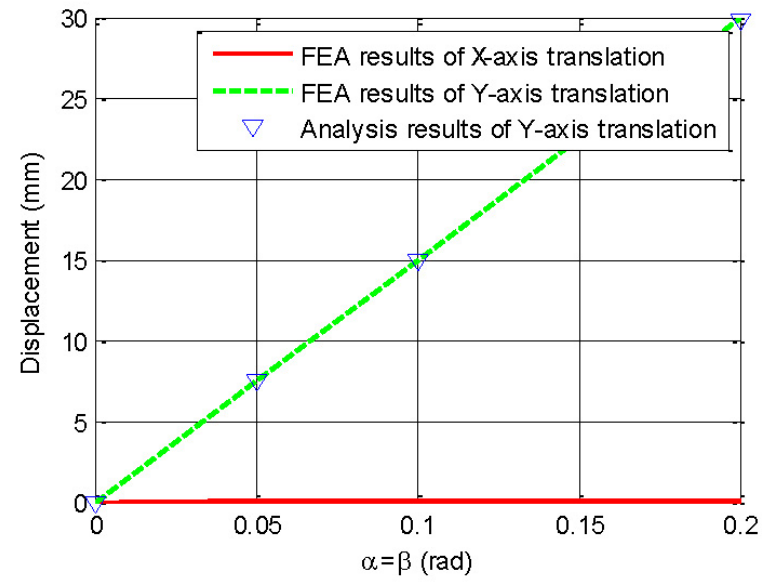

(a) Translations along the $\mathrm{X}$ and $\mathrm{Y}$ axes

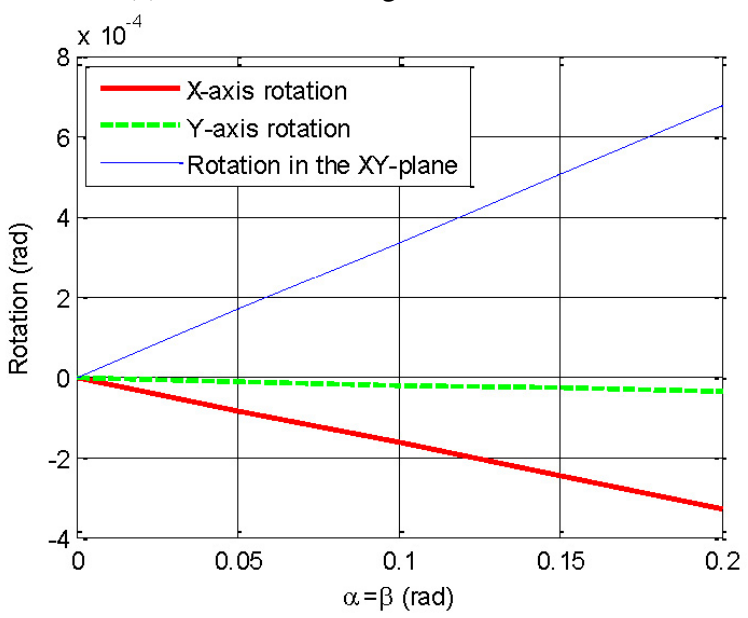

(b) Rotation about the Axis-L

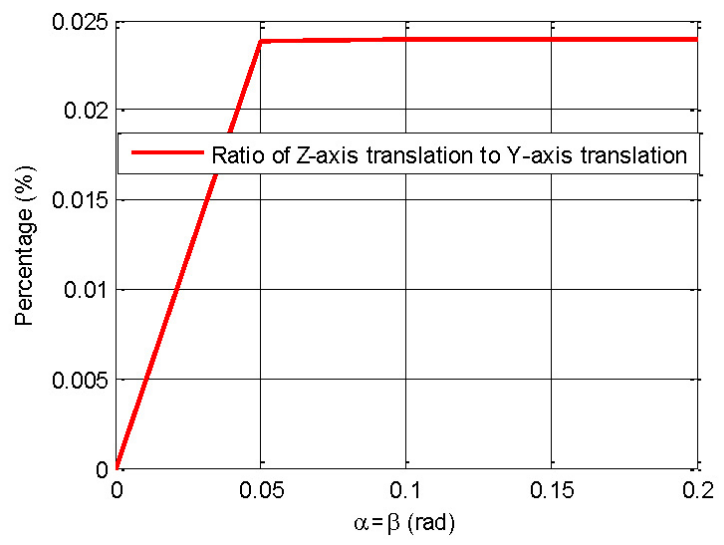

(c) parasitic motions (rotations about the $\mathrm{X}$ - and Y-axes and translation along the Z-axis)

FIGURE 13: FEA RESULTS FOR OPERATION MODE IV 
3. It can be seen from Figs. 10c, 11c, 12c, 13b and 13c that the parasitic motions are much smaller compared with the primary motions, for all the operation modes.

Overall, for all the operation modes of the compliant four-bar mechanism-2, the obtained analytical kinematic models are accurate enough to predict the rotation angle in the XY-plane and the translation displacement along the Y-axis, under specific input actuations. Additionally, the parasitic motions are much smaller than the primary motions, which ensures that the tiny effect of the parasitic motions on the primary motions can be ignored in an acceptable way. Therefore, it has been proved that the compliant four-bar mechanism- 2 can be operated in the different operation modes with high accuracy.

\section{A PROSPECTIVE APPLICATION AS A COMPLIANT GRIPPER}

The reconfigurable compliant four-bar mechanism-1 shown in Fig. 7 is used to design a reconfigurable gripper as shown in Fig. 14. It can exhibit four grasping modes based on the actuation of the linear actuator $1( \pm \alpha)$ or $2( \pm \beta)$ as displayed in Fig. 15. The first three grasping modes are angular, where the jaws of the gripper rotate about an instantaneous centre of rotation which is different for each grasping mode. The gripper displays an angular grasping mode when $\alpha \neq 0, \beta=0$ as shown in Fig. 15a, $\alpha=0, \beta \neq 0$ as shown in Fig. $15 \mathrm{~b}$ or when $\alpha<0, \beta<0$ as shown in the right Fig. 15c. The parallel grasping mode in which the jaws are parallel to one another is achieved when $\alpha>0, \beta<0$ as shown in the left Fig. 15c. Thus, the reconfigurable compliant gripper at hand unveils an ability to grasp a plethora of shapes unlike other compliant grippers in literature that exhibit only one of these modes of grasping [14, 19]. Potential applications include micromanipulation and grasping lightweight and vulnerable materials like glass, resins, porous composites, etc. in difficult and dangerous environments. In addition, it can be used for medical applications to grasp and manipulate living tissues during surgical operations or as a gripper mounted on a parallel manipulator dedicated to fast and accurate pick-and-place operations. Figure 16 shows the prototype of the reconfigurable compliant gripper.

\section{CONCLUSIONS AND FUTURE WORK}

A novel idea of designing mobility-reconfigurable compliant mechanisms inspired by the constraint singularities of rigid body mechanisms was presented in this paper. A rhombus planar rigid four-bar mechanism was analyzed to identify its three operation modes and two constraint singularities separating those modes. The rigid joints were replaced by compliant joints to obtain two designs of a reconfigurable compliant four-bar mechanism. The second design was found to be more accurate and
- Lumped compliance revolute joint

Instantaneous rotation centre of leaf-type isosceles trapezoidal joint

Grounded hole

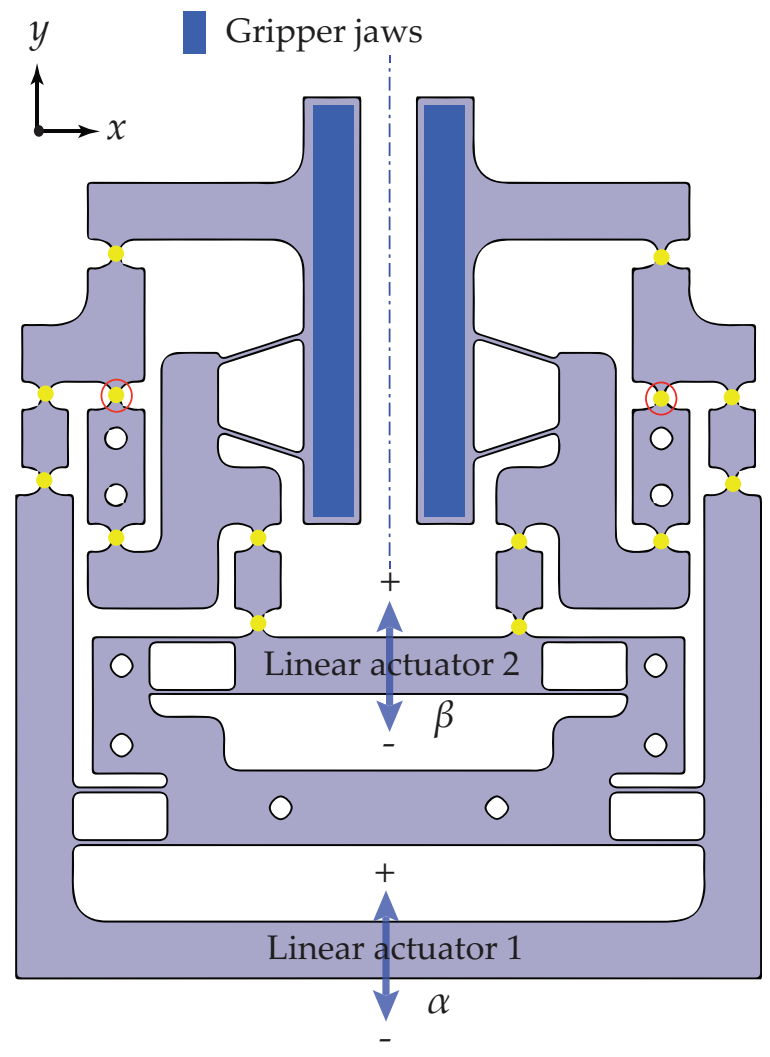

FIGURE 14: A novel reconfigurable compliant gripper

less parasitic than the first one, which is verified by its nonlinear FEA simulations in different motion modes. Moreover, the compliant four-bar mechanism was shown to have four operation modes based on the particular actuation strategy unlike its rigid counterpart. A preliminary design of a compliant gripper has been designed based on the reconfigurable compliant fourbar mechanism introduced and studied in this paper.

In the future, we will focus on the analytical kinetostatic modelling of the reconfigurable compliant mechanism at hand while exploring appropriate applications. We also intend to design mobility-reconfigurable compliant mechanisms based on the constraint singularities of spatial rigid body mechanisms.

\section{ACKNOWLEDGMENT}

The authors would like to express their gratitude for the Ulysses 2016 grant between Ireland and France. Mr. Tim Powder and Mr. Mike O'Shea in University College Cork are appreciated 

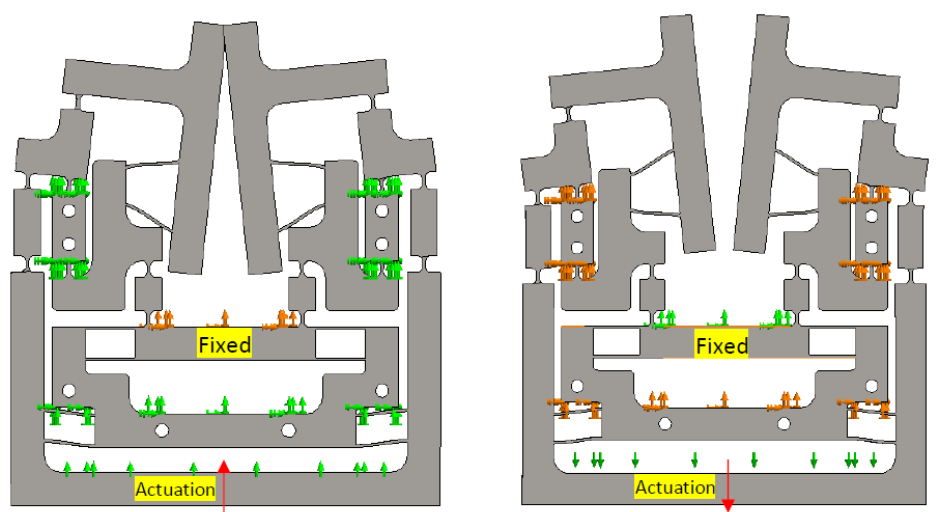

(a) Angular grasping mode $1: \alpha \neq 0, \beta=0$
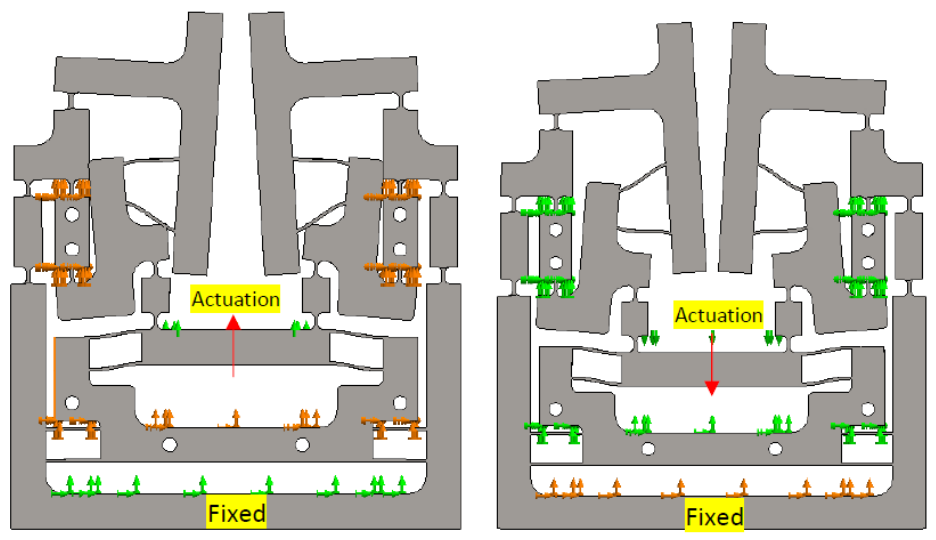

(b) Angular grasping mode $2: \alpha=0, \beta \neq 0$
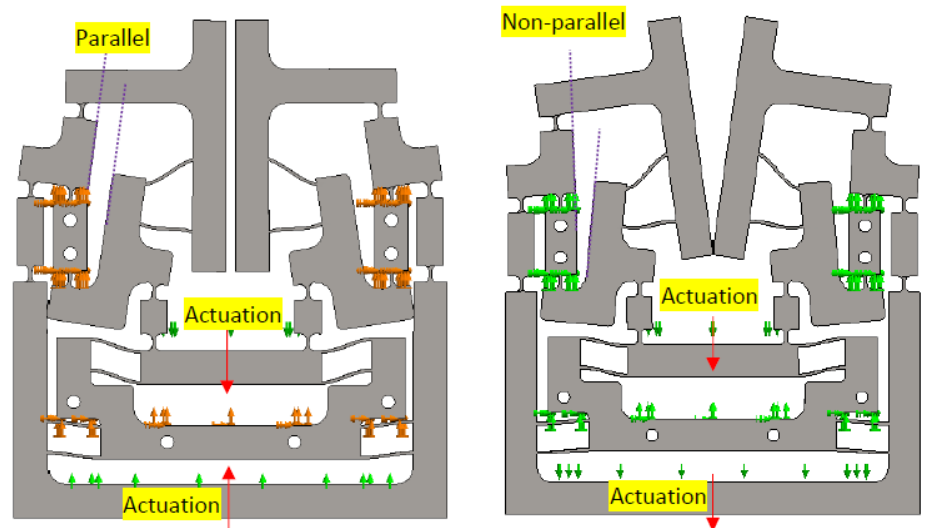

(c) Left : parallel grasping mode $(\alpha>0, \beta<0)$; Right : angular grasping mode $3(\alpha<0, \beta<0)$

FIGURE 15: FOUR GRASPING MODES OF THE COMPLIANT GRIPPER

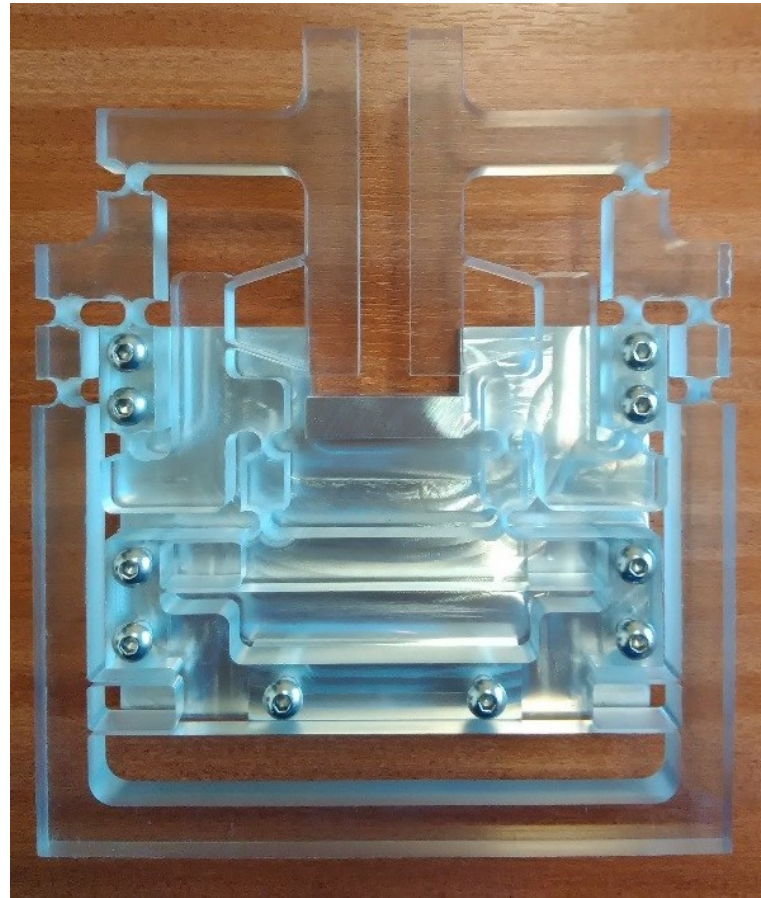

FIGURE 16: Prototype of the reconfigurable compliant gripper

for their kind help in the 3D printed prototype.

\section{REFERENCES}

[1] Gallego, J. A., and Herder, J., 2009. "Synthesis methods in compliant mechanisms: An overview.". In ASME. International Design Engineering Technical Conferences and Computers and Information in Engineering Conference, Vol. 7 of 33rd Mechanisms and Robotics Conference, Parts A and B. pp. 193-214.

[2] Olsen, B. M., Issac, Y., Howell, L. L., and Magleby, S. P., 2010. "Utilizing a classification scheme to facilitate rigid-body replacement for compliant mechanism design.”. In ASME. International Design Engineering Technical Conferences and Computers and Information in Engineering Conference, Vol. 2 of 34th Annual Mechanisms and Robotics Conference, Parts A and B. pp. 475-489.

[3] Howell, L. L., 2001. Compliant mechanisms.

[4] Hao, G., and Li, H., 2015. "Conceptual designs of multidegree of freedom compliant parallel manipulators composed of wire-beam based compliant mechanisms". Proceedings of the Institution of Mechanical Engineers, Part C: Journal of Mechanical Engineering Science, 229(3), pp. 538-555.

[5] Amine, S., Mokhiamar, O., and Caro, S., 2017. "Classification of 3T1R parallel manipulators based on their wrench 
graph”. ASME. J. Mechanisms Robotics., 9(1).

[6] Maraje, S., Nurahmi, L., and Caro, S., 2016. "Operation modes comparison of a reconfigurable 3-PRS parallel manipulator based on kinematic performance.". In Proceedings of the ASME 2016 International Design Engineering Technical Conferences and Computers and Information in Engineering Conference. pp. 21-24.

[7] Nurahmi, L., and Caro, S., 2015. "Dimensionally homogeneous extended jacobian and condition number.". In The 2nd International Conference on Mechanical Engineering (ICOME 2015). pp. 3-5.

[8] Rubbert, L., Caro, S., Gangloff, J., and Renaud, P., 2014. "Using singularities of parallel manipulators for enhancing the rigid-body replacement design method of compliant mechanisms". ASME Journal of Mechanical Design, 136, pp. 051010-1-051010-9.

[9] Rubbert, L., Renaud, P., Caro, S., and Gangloff, J., 2014. "Design of a compensation mechanism for an active cardiac stabilizer based on an assembly of planar compliant mechanisms". Mechanics \& Industry, 15(2), pp. 147-151.

[10] Zlatanov, D., Bonev, I. A., and Gosselin, C. M., 2002. Constraint Singularities as C-Space Singularities. Springer Netherlands, Dordrecht, pp. 183-192.

[11] Husty, M. L., Pfurner, M., Schröcker, H.-P., and Brunnthaler, K., 2007. "Algebraic methods in mechanism analysis and synthesis". Robotica, 25(6), Nov., pp. 661-675.

[12] Nurahmi, L., Caro, S., Wenger, P., Schadlbauer, J., and Husty, M., 2016. "Reconfiguration analysis of a 4-RUU parallel manipulator". Mechanism and Machine Theory, 96, Part 2, pp. $269-289$.

[13] He, X., Kong, X., Hao, G., and Ritchie, J., 2016. Design and Analysis of a New $7 R$ Single-Loop Mechanism with $4 R$, $6 R$ and $7 R$ Operation Modes. Springer International Publishing, Cham, pp. 27-37.

[14] Hao, G., and Hand, R. B., 2016. "Design and static testing of a compact distributed-compliance gripper based on flexure motion". Archives of Civil and Mechanical Engineering, 16(4), pp. $708-716$.

[15] Hao, G., Li, H., and Kavanagh, R. C., 2016. "Positionspace-based compliant mechanism reconfiguration approach and its application in the reduction of parasitic motion.”. ASME. J. Mech. Des., 138(9), pp. 092301-09230113.

[16] Li, H., and Hao, G., 2015. "Compliant mechanism reconfiguration based on position space concept for reducing parasitic motion.”. In ASME. International Design Engineering Technical Conferences and Computers and Information in Engineering Conference, Vol. 5A of 39th Mechanisms and Robotics Conference. pp. 475-489.

[17] Bottema, O., and Roth, B., 2012. Theoretical Kinematics. Dover Books on Physics. Dover Publications.

[18] Zhao, H., Bi, S., and Yu, J., 2012. "A novel compliant linear-motion mechanism based on parasitic motion compensation". Mechanism and Machine Theory, 50, pp. 15 28.

[19] Hao, G., and Kong, X., 2012. Conceptual design and modelling of a self-adaptive compliant parallel gripper for highprecision manipulation. 